\title{
Stable Partitions in a Model with Group-Dependent Feasible Sets*
}

\author{
Michel Le Breton ${ }^{\dagger} \quad$ Shlomo Weber ${ }^{\ddagger}$
}

May 2003

\begin{abstract}
In this paper we consider a model of group formation where group of individuals may have different feasible sets. We focus on two polar cases, increasing returns, when the set of feasible alternatives increases if a new member joins the group, and decreasing returns, when a new member has an opposite effect and reduces the number of alternatives available for the enlarged group. We consider two notions, stability and strong stability of group structures, that correspond to Nash and Strong Nash equilibrium of the associated non-cooperative game. We prove existence results for various classes of environments and also investigate the link between the dimensionality of the set of alternatives and the existence of stable structures.
\end{abstract}

Keywords: Feasible sets, Stable partitions, Positive externality, Increasing and Decreasing Returns.

JEL Classification Numbers: C71, C72, D62, D71.

${ }^{*}$ The last version of this paper has been written while the second author was visiting the Technical University of Dresden as the Humboldt Research Prize Holder. He wishes to thank the Alexander von Humboldt Foundation for its generous support and the Technical University of Dresden for its hospitality.

†Université de Toulouse I, GREMAQ and IDEI, Toulouse, France.

$\ddagger$ Department of Economics, Southern Methodist University, Dallas, USA and CORE, Catholic University of Louvain-la-Neuve, Belgium. 


\section{Introduction}

In this paper we consider a model with heterogeneous individuals who have preferences over a given set of alternatives and partnerships. They partition themselves into groups (coalitions), where each group chooses an alternative from its own feasible set. Every individual benefits from the large size of the group she belongs to, however the choice of an alternative by that group could be detrimental for some of its members. In this regard our model related is to the brand of literature on coalition formation that attempts to resolve the conflict between increasing returns to scale in large groups and heterogeneity of individual preferences. Indeed, it might be beneficial to be a member of a large political party due potential benefits and perks of power. However, the structure of a large party could be such that its political platform chosen by its committees or conventions might be very distant from the preferences of some of the party members. In this case the party unity could be under threat of loosing a part of its membership, who may even create their own party. The question that may arise is whether the breakaway group has sufficient resources and a power of conviction to survive on its own.

In general, the problem of group stability is rooted in the comparative power of small versus large groups. In this paper we examine this aspect of stability of group formation by focusing our attention at the set of feasible options available for groups of individuals when they form. The main distinctive feature of our model is that groups of individuals, when they form, have different feasible sets. Most of the existing literature (Guesnerie and Oddou (1879, 1981, 1988), Greenbeg and Weber (1986), Demange (1994),Demange and Guesnerie (1997), Konishi, Le Breton and Weber(1997,a-e), among others $)^{1}$ consider the case where the set of available choice for all coalitions is the same. However, one may consider various situations where this assumption does not necessarily hold. For example, a merger of two firms, may generate technological and market opportunities that were not available in the pre-merger

\footnotetext{
${ }^{1}$ Greenberg and Weber (1993) is an exception.
} 
environment, and the firm would benefit from increasing returns to scale. The enlargement of the European Union is often supported by the claim that the whole is larger than the sum of its parts. Thus, the enlarged union offers its new members prospects and opportunities that are unavailable outside of the union. On the other hand, in the framework of an international conflict, presence of a large number of participants could make it difficult to find a policy acceptable for all parties involved. Even though everybody would prefer a joint action, finding a compromise becomes increasingly difficult when the number of participants is large. In this case one may assume that the set of feasible, or acceptable, alternatives is a declining function of the group size. The same situation may occur in business, political or any kind of negotiations where each of participants has a predetermined set of acceptable alternatives. Thus, the question would be whether it is possible to find a suitable compromise that would be unanimously accepted, and the large number of heterogeneous participants can create difficult barriers to overcome.

We also relate our results on stability of group formation to the number of dimensions of the alternative space. It is quite natural to expect that the ability to reach a compromise among various participants is crucially dependent on the dimensionality of the conflict. In general, the severity of the conflict raises when the number of its dimensions increases, in which case the search for a stable group structure that would satisfy all participants becomes more challenging.

We consider two extreme cases, increasing returns, when the set of feasible alternatives increases if a new member joins the group, and decreasing returns, when a new member has an opposite effect and reduces the number of alternatives available for the enlarged group. We associate our model of group formation with a noncooperative game in strategic form and examine the notions of Nash equilibrium and strong Nash Equilibrium of this game that correspond to stability and strong stability of group structures. We then identify the classes of environments that admit either stable or strongly stable group structures under 
increasing or decreasing returns. We also investigate how the stability is linked to the number of dimensions of the alternative set examine the case of dichotomy where all groups are divided in two types, effective, whose feasible set is the entire alternative set, and ineffective, whose feasible sets are empty. It turns out that the Nakamura number plays an important role in our investigation.

The paper is organized as follows. In the next section we describe the model and introduce the assumptions that will be used to prove our results. In Section 3 we define the noncooperative and cooperative games associated with our model and determine the link between them. Section 4 is devoted to the discussion on the impact of the dimensionality of the set of alternatives on stability of group structures. In Sections 5 and 6 we state our existence results in the case of increasing and decreasing returns, respectively. The proofs of the results are relegated to the Appendix.

\section{The Model}

In our setting, a society (environment) $E$ is defined as a quadruple $(N, \Omega, \Phi, U)$, where $N=\{1, \ldots, n\}$ is a set of players, $\Omega$ is a set of alternatives, $\Phi$ is a feasibility correspondence that assigns the set $\phi(S) \subset \Omega$ to every coalition $S \subset N$, and $U=\left\{u_{i}\right\}_{i \in N}$ is a vector of players utility functions that represent players' preferences over pairs of alternatives and coalitions: $u_{i}: \Omega \times \mathcal{S}^{i} \rightarrow \Re_{+}$, where $\mathcal{S}^{i}$ denotes the set of coalitions that contain $i$.

The agents may form different groups (coalitions). Each coalition $S$ has a set of feasible alternatives $\phi(S) \subset \Omega$; thus, if coalition $S$ forms, it can choose an alternative from $\phi(S)$. The notion of feasibility may have different interpretations. If the alternatives under consideration are allocations of goods, feasibility, as usual, means that, due to resource constraints, some alternatives may be out of reach for some individuals or coalitions. But feasibility may also incorporate constraints that are generated by institutions, rules or social norms. These second best constraints limit further the scope of action of coalitions. Such a 
broad interpretation allows us to examine the feasibility correspondence in a wide range of environments.

We impose two assumptions that hold throughout the rest of the paper. One requires that for every player it is better to be a member of any coalition that chooses a feasible alternative rather than to be a member of a coalition that chooses an alternative outside of its feasible set. Thus, if possible, every individual would prefer to join a coalition choosing a feasible alternative rather that staying in the group that chooses an alternative outside its feasible set.

Desirability of feasible alternatives - DFA: For every $i \in N$, two coalitions $S, S^{\prime} \in \mathcal{S}^{i}$, and two alternatives $\omega \in \phi(S), \omega^{\prime} \notin \phi\left(S^{\prime}\right)$, we have $u_{i}(\omega, S)>u_{i}\left(\omega^{\prime}, S^{\prime}\right)$.

The notion of desirability is introduced through the definition of $u_{i}(\omega, S)$ by assigning very low values to all $u_{i}(\omega, S)$ for which $\omega \notin \phi(S)$. Similarly, the fact that $\phi(S)=\emptyset$ for some coalition $S$ can be interpreted as meaning that for every player in $i$ in $S$, each feasible alternative in other coalition would yield $i$ a higher payoff than one she can obtain within $S$. In short, we want any group of players to rule out any alternative outside of their but in order to formally define a non-cooperative game below, we have to assign a (unacceptably low) value to any alternative outside of the group's feasible set.

We also require that the utility of a player within every coalition would not decline if a new member joins that coalition without altering the chosen alternative.

Positive Externality - PE: For every $i \in N$, every two coalitions $S \subset S^{\prime}$ with $S \in \mathcal{S}^{i}$, and every $\omega \in \phi(S) \bigcap \phi\left(S^{\prime}\right)$, we have $u_{i}(\omega, S) \leq u_{i}\left(\omega, S^{\prime}\right)$.

In addition to these two basic assumptions, we introduce some additional requirements that we need for some of our results. In the case of unidimensional set of alternatives, the convexity of preferences corresponds to the classical property of single-peakedness that yields the existence of a top alternative for each player within a given coalition, such that her utility 
would decline the further away she is from her ideal choice. It order to avoid some technical problems, we often turn to a finite set of alternatives.

Single-peakedness - SP: $\Omega$ is a finite subset of the real line and for every $i \in N$ and $S \in \mathcal{S}^{i}, u_{i}(\cdot, S)$ is single-peaked on $\phi(S)$.

The next property, labelled anonymity, implies that the utility of each individual depends only on the number but not the identity of other members of the coalition she is in.

Anonymity - AN: For every $i \in N$, every two coalitions $S, S^{\prime} \in \mathcal{S}^{i}$ with $|S|=\left|S^{\prime}\right|$, and every $\omega \in \phi(S) \cap \phi\left(S^{\prime}\right)$, we have $u_{i}(\omega, S)=u_{i}\left(\omega, S^{\prime}\right)$.

The class of environments that satisfy $\mathrm{DFA}, \mathrm{PE}, \mathrm{SP}$ and $\mathrm{AN}$ is denoted by $\mathcal{E}$. In some cases, we consider a stronger assumption:

Separability - SEP: $\Omega$ is a finite subset of the real line and for every $i \in N$, the utility function $u_{i}$ can be represented $\mathrm{as}^{2}$

$$
u_{i}(\omega, S)=\left\{\begin{array}{lll}
v_{i}(\omega) & \text { if } \omega \notin \phi(S) \\
v_{i}(\omega)+h(|S|) & \text { if } \quad \omega \in \phi(S)
\end{array}\right.
$$

where $v_{i}(\cdot)$ is single-peaked over $\phi(S)$, and $h(\cdot)$ is strictly increasing on the set of positive integers. (Note that the function $h$ does not depend on $i$ ).

To guarantee that the desirability of feasible alternatives assumption holds, we will require that $h(1)>v_{i}(\omega)-v_{i}\left(\omega^{\prime}\right)$ for every $i \in N, \omega, \omega^{\prime} \in \Omega$. The class of environments that satisfy SEP is denoted by $\mathcal{E}^{\prime}$.

Sometimes we employ a stronger assumption than separability. Namely, we require that every individual has an ideal alternative $\omega^{i}$, such that her utility function is represented by the Euclidean distance function from alternative $\omega$ to $\omega^{i}$.

\footnotetext{
${ }^{2}$ Konishi and Fishburn (1996) provide an axiomatic characterization of separable preferences.
} 
Euclidean preferences: Suppose that for every $i$ there exists a unique peak, $\omega^{i}$, such that the utility function $u_{i}$ is given by

$$
u_{i}(\omega, S)= \begin{cases}-\left|\omega-\omega^{i}\right| & \text { if } \omega \notin \phi(S) \\ -\left|\omega-\omega^{i}\right|+h(|S|) & \text { if } \omega \in \phi(S)\end{cases}
$$

where, again, $h(\cdot)$ is strictly increasing on the set of positive integers, and for every $i \in N, \omega, \omega^{\prime} \in \Omega, h(1)>v_{i}(\omega)-v_{i}\left(\omega^{\prime}\right)$.

The class of environments that satisfy EP is denoted by $\mathcal{E}^{\prime \prime}$. We have the following inclusion:

$$
\mathcal{E}^{\prime \prime} \subset \mathcal{E}^{\prime} \subset \mathcal{E} .
$$

Denote by $\mathcal{E}_{l}$ the classes of environments that belong to $\mathcal{E}$ and satisfy Assumption $l=$ $I R, D R$. Similar notation is used for $\mathcal{E}^{\prime}$ and $\mathcal{E}^{\prime \prime}$.

As we mentioned in the introduction, the crucial role in our analysis will be played by the feasibility correspondence. In particular, we distinguish between two cases: one, when every new member of a coalition expands its feasible set, and another, when an entry of a new member shrinks the existing feasible set. ${ }^{3}$ Formally,

Increasing Returns - IR: For every two coalitions $S \subset S^{\prime}$ we have $\phi(S) \subset \phi\left(S^{\prime}\right)$.

Decreasing Returns -DR: For every two coalitions $S \subset S^{\prime}$ we have $\phi\left(S^{\prime}\right) \subset \phi(S)$.

Occasionally we will mention the case of constant returns which would simply describe the environments for which both Assumption $I R$ and $D R$ hold. This simply means that all nonempty coalitions have the same feasible set.

In the next section we introduce both non-cooperative and cooperative descriptions of players' interaction and establish an important link between two approaches.

\footnotetext{
${ }^{3}$ Intermediate cases, like those familiar in the theory of clubs where increasing returns can be bounded by congestion effects could be considered - see Konishi, Le Breton and Weber (1997b).
} 


\section{Non-cooperative and Cooperative Framework}

First, we consider a noncooperative game $\Gamma$ where the strategy set of each player is given by the set $\Omega$. Each player simultaneously and independently select an alternative. These choices determine a partition of the players into several groups to which we will refer hereafter as a group structure: two players are in the same group if they have selected the same alternative. A physical outcome consists of a group structure together with an alternative for each group. If every player $i$ chooses a strategy $\omega_{i}$ this gives rise to the strategy profile $x=\left(\omega_{1}, \ldots, \omega_{n}\right)$. For every $\omega \in \Omega$ denote by $N_{\omega}(x)$ the set of players who choose $\omega$ in $x$. Those choices generate a partition $P(x)$ of the set $N$ into pairwise disjoint sets $\left\{S_{1}, \ldots, S_{K}\right\}$ each consisting of the players choosing the same alternative in $x$. The payoff function of player $i$ is given by $u_{i}\left(\omega_{i}, N_{\omega_{i}}(x)\right)$, so that the payoff of player $i$ depends both on the alternative selected by that player and the subset of players selecting the same alternative.

An important feature of this formulation is the independence of the player's payoff with respect to choices different from hers. That is, whenever groups are formed, any change in one of them has no impact on the others as long their choices remain different. This important property has been studied under different names ( "orthogonality" in Guesnerie and Oddou (1988), "games without externalities" in Bloch (1996), or "games without spillovers" in Konishi, Le Breton and Weber (1997e)). It obviously rules out some important group formation problems like cartel or association agreements in industrial organization (Belleflamme (2000), Bloch (1995)), custom unions (Yi (1997)) and environmental agreements (Ray and Vohra (2001)), among others. To emphasize this feature ${ }^{4}$, we usually reserve the term group formation for this case in contrast to the terminology coalition formation, which covers any strategic setting where independent players have the opportunity to form

\footnotetext{
${ }^{4}$ It is worthwhile to remind that this assumption is implicit in all cooperative game theory where the use of the concept of characteristic function itself presumes the group's payoff is not affected by actions of its complement.
} 
coalitions and make bidding agreements.

It is worth to pointing out that an outcome of the game consists not only of a group structure but also of a vector of alternatives, where one alternatives is chosen by one group. These chosen alternatives play an important role in many cases. But there are environments where these alternatives do not play a direct role and simply represent, as mentioned above, coordination devices. These games correspond to the particular case where the utility function $u_{i}(\cdot, \cdot)$ only depends only on the set of players choosing the same alternative but not on the alternative itself. Those so-called "hedonic games" ${ }^{5}$ games (Banerjee, Konishi and Sömnez (2001), Bogomolnaia and Jackson (2002), Milchtaich and Winter (2002)) ${ }^{6}$.

We now introduce two notions of equilibrium (or stability) that will be used in this paper. Consider, first, the notion of Nash equilibrium in pure strategies. In our framework, this notion imposes the following natural requirements. First, even if the set of feasible alternatives for individual $i$ is nonempty, she would not be better off by staying alone. Secondly, suppose that player $i$ contemplates joining another coalition $S$ in the existing partition $P(x)$ all members of which choose alternative $\omega$. If $\omega$ is not feasible for the coalition $S \cup\{i\}$, the move obviously would not take place. But even if the alternative $\omega$ is feasible for the coalition $S \cup\{i\}$, the move should not be beneficial for player $i$.

Definition 3.1: A strategy profile $x=\left(\omega_{1}, \ldots, \omega_{n}\right)$ is a Nash equilibrium if

(i) $\left.u_{i}\left(\omega_{i}\right), N_{\omega_{i}}(x)\right) \geq \max _{\omega \in \phi(\{i\})} u_{i}(\omega,\{i\})$ for every $i \in N$;

(ii) For every individual $i$ and every alternative $\omega \neq \omega_{i}$ with $N_{\omega}(x) \neq \emptyset$, either $\omega \notin$ $\phi\left(N_{\omega}(x) \bigcup\{i\}\right)$ or $u_{i}\left(\omega_{i}, N_{\omega_{i}}(x)\right) \geq u_{i}\left(\omega, N_{\omega}(x) \bigcup\{i\}\right)$.

The resulting partition $P(x)$ is called stable.

The notion of a strong Nash equilibrium (Aumann (1959)) is more demanding. It requires

\footnotetext{
${ }^{5}$ The term was introduced in Drèze and Greenberg (1980. It captures the fact that every player in this group formation game cares about her partners in the group.

${ }^{6}$ This type of games also emerges in the sequential setting, where groups form first and then according to some mechanism (voting, social planner, market) each group selects an alternative. After solving the game backwards, we eliminate the second stage and end up, in essence, with a hedonic group formation game.
} 
that the profile of strategies is immune to any deviation by any coalition. There are no restrictions on the deviation except for the fact that it must be profitable to all the members of the deviating coalition ${ }^{7}$. In our context, a strategy profile is a strong Nash equilibrium if there exists

no coalition, which is not necessarily an element of the existing partition, that possesses a feasible alternative that is beneficial for all its members. Strong Nash equilibrium is an appealing concept that solves an array of coordination problems. However, given its stringent requirements, there are not many environments that admit the existence of a strong Nash equilibrium. Some classes of environments, for which it does exist, are indicated below. Formally,

Definition 3.2: A strategy profile $x=\left(\omega_{1}, \ldots, \omega_{n}\right)$ is a strong Nash equilibrium if there is no coalition $S \subset N$ and a profile $x^{\prime}=\left(\omega_{1}^{\prime}, \ldots, \omega_{n}^{\prime}\right)$ with $\omega_{i}^{\prime}=\omega_{i}$ for all $i \notin S$ such that $u_{i}\left(\omega_{i}^{\prime}, N_{\omega_{i}^{\prime}}\left(x^{\prime}\right)\right)>u_{i}\left(\omega_{i}, N_{\omega_{i}}(x)\right)$ for all $i \in S$.

The resulting partition $P(x)$ is called strongly stable.

Let us now describe a cooperative variant of our model and associate a cooperative game in characteristic form $v$ with the noncooperative game of group formation described above. The characteristic function of game $v$ yields the value $v(S)$ for every $S \subset N$ which is determined by:

$$
v(S)=\left\{u \in \Re^{n}: \exists \omega \in \phi(S) \text { s.t. } u_{i} \leq u_{i}(\omega, S) \forall i \in S\right\}
$$

We use the standard definition of the core:

Definition 3.3: A vector of payoffs $u$ is in the core of the game $v$ if $u \in V(N)$ and there is coalition $S \subseteq N$ and $u^{\prime} \in v(S)$ such that $u_{i}^{\prime}>u_{i}$ for all $i \in S$.

\footnotetext{
${ }^{7}$ One may examine an alternative concept of coalition-proof Nash equilibrium (Bernheim, Peleg and Whinston (1987), ) that aims to introduce robustness with respect to potential coalitional deviations. While, in general, strong Nash equilibria and coalition-proof Nash equilibria yield different sets of equilibria, the two sets coincide for a large class of group formation games (see Konishi, Le Breton and Weber (1997c)).
} 
Denote by $\mathcal{P}$ the set of all partitions or group structures ${ }^{8}$ of $N$ and consider a partition $P=\left(S_{k}\right)_{1 \leq k \leq K} \in \mathcal{P}$. Then the set of feasible payoffs would be given by the following intersection:

$$
v(P)=\cap_{1 \leq k \leq K} v\left(S_{k}\right) .
$$

This allows us to define the superadditive cover $\widehat{v}$ of the game $v$ :

$$
\widehat{v}(S)=\cup_{P_{S} \in \mathcal{P}(S)} \cap_{T \in P_{S}} v(T)
$$

for all $S \subseteq N$, where $\mathcal{P}(S)$ denotes the set of partitions of $S$. The game $\widehat{v}$ describes the set of payoffs feasible for that coalition when the group can be partitioned in any arbitrary way. The games $v$ and $\widehat{v}$ coincide if $v$ is superadditive, where, to recall, the game $v$ is superadditive if $v(T) \cap v(S \backslash T) \subseteq v(S)$ for every $S \subseteq N$ and every $T \subseteq S$.

Since an element is in the core only if it is associated with an outcome immune against coalitional deviation, it follows that when $v$ is not superadditive, the definition of core has to be modified. Following Guesnerie and Oddou (1979), the natural way to proceed is to consider payoff vectors in the core of $\widehat{v}$. The following proposition states the connection between the noncooperative group formation game $\Gamma$ and the cooperative game $\widehat{v}$ :

Proposition 3.4: Let $E$ be a society satisfying DFA,PE and IR. The strategy profile $x=$ $\left(\omega_{1}, \ldots, \omega_{n}\right)$ is a strong Nash equilibrium of $\Gamma$ if and only if the vector $u \in \Re^{n}$, where $u_{i}=u_{i}\left(\omega_{i}, N_{\omega_{i}}(x)\right)$ for all $i \in N$, is in the core of $\widehat{v}$.

\section{Proof: See Appendix.}

Since the main goal of this paper is to investigate the existence of a Nash equilibrium and Strong Nash equilibrium in pure strategies in the case of heterogeneous feasible sets, the usefulness of Proposition 3.4 lies in the fact that it allows to import results from cooperative game theory to use them in the non-cooperative setting.

\footnotetext{
${ }^{8}$ Cooperative games with a coalition structure were introduced first by Aumann and Drèze (1974). We refer the reader to Greenberg (1994) for an excellent, though somewhat outdated, review of the literature on coalition structures.
} 


\section{On Dichotomy, Role of Dimensions and the Naka- mura Number}

In this section we review some existing results on the nature of the group(s) that would form at equilibrium and offer offering some observations on the role of the dimension or cardinality of the set of alternatives in the existence of a strong Nash equilibrium. Formally, we consider two alternative assumptions, Convexity and Finiteness:

Convexity - CNV: $\Omega$ is a subset of $\Re^{m}$. For every group $S$ the feasible set $\phi(S)$ is a convex subset of $\Re^{m}$ and for every $i \in S$, the utility function $u_{i}(\cdot, S)$ is quasi-concave on $\phi(S)$.

Alternatively, we consider a finite variant of the model:

Finiteness - FIN: The set of alternatives $\Omega$ is a finite set.

In terms of applications, one can easily construct environments where one of the variants (finite or continuous) provides a more appropriate description of the problem.

Under the assumption of positive externality and increasing returns, there are good reasons for the grand coalition $N$ to form. However, it is not difficult to construct societies for which this is not true. As already alluded to, this has to do with the severity of the potential conflicts among the individuals. When groups have to reach a consensus on multidimensional issues, it is natural to expect that the larger the dimension $m$ (or the cardinality of the set of alternatives $\Omega$ in the finite case), the more difficult it would be to find such a consensus. Thus, these two values could be considered measures of the severity of the conflict.

We start with the notion of an efficient outcome:

Definition 4.1: An efficient outcome is a vector $u \in \Re^{n}$ such that

(i) there is group structure $P \in \mathcal{P}$ for which $u \in v(P)$,

(ii) there is no other group structure $P^{\prime}$ and $u^{\prime} \in v\left(P^{\prime}\right)$ such that $u_{i}^{\prime}>u_{i}$ for all $i \in N$. A group structure $P$ is efficient is there is an efficient outcome $u \in v(P)$. A group structure $P$ is universally efficient if any efficient outcome $u \in v(P)$. 
Guesnerie and Oddou (1988) have introduced the following general condition on the profile of utility functions:

Definition 4.2: A profile of utility functions $U=\left\{U_{i}\right\}_{i \in N}$ satisfies the condition of $(q+1)$ multilateral agreements in $N$ if for any group structure $P=\left(S_{1}, \ldots, S_{K}\right)$, for any $u \in v(P)$ and any group $T$ with $q+1$ members there exists an alternative $\omega \in \phi(N)$ such that $u_{i} \leq U_{i}(\omega, N)$ for all $i \in T$.

Proposition 4.3 (Guesnerie and Oddou (1988): Let $E$ be a society satisfying DFA, $\mathrm{PE}$ and CNV. If $n>m$, then $N$ is universally efficient if and only if the profile of utility functions satisfies the condition of $(m+1)$ multilateral agreements.

We have already pointed out that the superiority of large groups over smaller ones relies on ability of members of a large group to reach an unanimously acceptable and beneficial compromise. It may be quite difficult and demanding to check for all possible proposals and the main contribution of Proposition 4.3 is to limit the verification of the conditions to a proper subset of coalitions; the smaller is the number $m$, the smaller is the subset of coalitions to be examined. In particular, in the unidimensional case the condition of $(m+1)$ multilateral agreements corresponds to a condition of bilateral agreements which can be formulated as follows:

For any two individuals $i, j \in N$, for any group $S$ with $i \in S$ and $j \in N \backslash S$, there exists an alternative $\omega \in \phi(N)$ such that

$$
\max _{\omega^{\prime} \in \phi(S)} U_{i}\left(\omega^{\prime}, S\right) \leq U_{i}(\omega, N) \text { and } \max _{\omega^{\prime} \in \phi(N \backslash S)} U_{j}\left(\omega^{\prime}, N \backslash S\right) \leq U_{j}(\omega, N \backslash S)
$$

The condition implies that if the entire population is divided into two groups and if individuals $i$ and $j$ were selected to act as dictators in their respective groups, then these two individuals should be able to find a compromise when considering to merge their groups. It is important to note that the condition of bilateral agreements requires a finite number of 
inequalities to be checked, which is not the case in the multidimensional framework. Hence, in the unidimensional setting Proposition 4.3 formalizes and simplifies the intuitive notion of a profile of preferences that exhibit a limited degree of conflict.

The above result examines the universal efficiency of the grand coalition and does not provide any information of its stability ${ }^{9}$, and moreover, it does not consider alternative group structures. To offer some results in these directions, we need the following definition:

Definition 4.4: A feasible correspondence $\phi$ is dichotomic if it satisfies IR and for every $S \subseteq N$ either $\phi(S)=\Omega$ (effective group) or $\phi(S)=\emptyset($ ineffective $)$

Dichotomic feasible correspondences represent a specific class among feasible correspondences displaying increasing returns: every group is either completely effective or completely ineffective. While extreme, this discontinuous form of increasing returns to size occurs in many important examples. Let $\mathcal{C}$ be the family of effective coalitions, i.e., $S \in \mathcal{C}$ if and only if $\phi(S)=\Omega$.

Definition 4.5: Let the family of efficient coalitions $\mathcal{C}$ be given. Let $A(\mathcal{C})$ be the set of integers defined by

$$
A(\mathcal{C}) \equiv\left\{K \mid \exists \text { groups } S_{1}, \ldots, S_{K} \in \mathcal{C} \text { such that } \cap_{1 \leq k \leq K} S_{k}=\emptyset\right\}
$$

The Nakamura number of the family $\mathcal{C}$ is the integer $\nu(\mathcal{C})$ defined by

$$
\nu(\mathcal{C})=\left\{\begin{array}{lll}
\min \{K \mid K \in A(\mathcal{C})\} & \text { if } & A(\mathcal{C}) \neq \emptyset \\
+\infty & \text { if } & A(\mathcal{C})=\emptyset
\end{array}\right.
$$

This integer, introduced by Nakamura (1979) to study voting in committees, provides a useful combinatorial information about the effective groups and the nature of the increasing returns to size described by the feasible correspondence $\phi$. If returns to size are already

\footnotetext{
${ }^{9}$ In the specific second best taxation problem considered by Guesnerie and Oddou (1981), it turns out that universal efficiency and strong stability coincides. A proof based on balancedness is provided in Greenberg and Weber (1982). Some results are also contained in Demange and Guesnerie (1997).
} 
exhausted by relatively small coalitions, the Nakamura number is likely to be small and it would become more difficult to obtain a stable group structure. In what follows, we denote by $\nu(\phi)$ the Nakamura number of the family of effective coalitions induced by the feasible correspondence $\phi$.

Proposition 4.6: Let $E$ be a society satisfying DFA, PE, CON and DIC. Suppose also that the family $\mathcal{C}$ satisfies exclusive effectiveness: $S \in \mathcal{C}$ implies $N \backslash S \notin \mathcal{C}$. Then the grand coalition $N$ is universally efficient. If, moreover, $m \leq \nu(\phi)-2$, it is also strongly stable.

Proof: See Appendix.

It is possible to show that if $m \geq \nu(\phi)-1$ and there are too many dimensions in the decision problem (given groups' feasible sets), then there exist societies without a strongly stable group structure. The exclusive effectiveness in Proposition 4.6 rules out a possibility that each of two disjoint groups has the same feasibility range as the grand coalition. In some situations, the power of smaller groups may enhance efficiency as the group structure can contain several groups making their own choices. However, in presence of many small powerful groups, stability is more difficult to obtain.

It is worth to point out that replacing convexity by finiteness in Proposition 4.6, will generate similar results. The condition $m \leq \nu(\phi)-2$ should simply be replaced by the condition $|\Omega| \mid \leq \nu(\phi)-1$. Under the finiteness assumption the relation between the existence of stable structures and the cardinality of the feasible set has been studied by Deb, Weber and Winter (1996). They examined the class of quota games, where $S$ is effective if and only if its size reaches an integer threshold $q$ and, obviously, if $q \leq \frac{n}{2}$, the exclusive effectiveness is violated. Deb, Weber and Winter have explicitly calculated the integer $\rho(\phi)$ such that if $|\Omega| \leq \rho(\phi)-1$, then there exists a strongly stable group structure.

We are not aware of a study of the general case in the continuous and convex setting, but we could conjecture that a result similar to Proposition 4.6 can be obtained. However, if $m \geq 2$, then there exist societies satisfying the convexity assumption for which there are 
no strongly stable group structure. In this context, Le Breton and Weber (1995) considered the case where $q=2, \Omega=\Re^{2}$ and $U_{i}(\omega, S)=-\left\|\omega-\theta_{i}\right\|$ for all $i \in N$ and all $S \subseteq N$ where $\theta_{i} \in \Re^{2}$ is the "ideal" point of individual $i$. They provide a necessary and sufficient condition on the matrix of pairwise distances between ideal points to guarantee the existence of a strongly stable group structure. The characterization provides information about the nature and the magnitude of the disagreement between individual preferences leading to a strongly stable coalition structure despite the fact that very small coalitions are very powerful. But the question of a general characterization of the class of societies for which a strongly stable coalition structure exists remains open.

Finally, in this dichotomic setting, there are environments where the feasible set of a coalition is not so much related to the number of individuals in the coalition but to the joint characteristics (or types) of the individuals in the coalition. A complete characterization of the families $\mathcal{C}$ for which a strongly stable group structure always exists is provided in Kaneko and Wooders (1982) and Owen, Le Breton and Weber (1992).

One lesson from the results obtained in the dichotomic case is that the existence of a strongly stable coalition structure is intimately related to a comparison between a combinatorial index summarizing the dispersion of power across potential groups and the dimension (or the cardinality) of the set of alternatives. It has been observed that when alternatives are described by more than one dimension, it becomes very difficult to ensure existence: for instance, when $m=2$ and feasibility only depends on the number of individuals in the group, groups with less than $\frac{2}{3}$ of the entire population must be powerless.

Those insights raise a number of open problems. Is it possible to obtain similar results in a nondichotomic setting? For instance, it would be interesting to examine the case with three types of groups, effective, ineffective, and intermediate, whose feasible set is a given subset of $\Omega$. Furthermore, we have focused here on the existence of strongly stable group structures but one could examine stable structures as well. Some partial answers to these 
questions are provided in the next two sections.

\section{$5 \quad$ Increasing Returns}

Now, let us turn to the case of increasing returns. Without loss of generality, let $\phi(N)$ be the entire nonempty set $\Omega$. The case of increasing returns has attracted most of the attention but very few general results are available. Within the class $\mathcal{E}$, Greenberg and Weber (1993) show that strong Nash equilibria always exist if for all $i \in N, \omega \in \Omega$ and for any two coalitions $S, T \in \mathcal{S}^{i}, u_{i}(\omega, S)=u_{i}(\omega, T)$ as long as $\omega$ is feasible for both $S$ and $T$. Within the class $\mathcal{E}^{\prime}$, Konishi, Le Breton and Weber (1997a) have proved that Nash equilibria always exist whenever $\phi$ exhibits constant returns to scale.

Our first example shows that within the class $\mathcal{E}_{I R}^{\prime}$, and therefore the class $\mathcal{E}_{I R}$, a Nash equilibrium may fail to exist.

Example 5.1 We construct a quadruple $E$ that belongs to $\mathcal{E}^{\prime}{ }_{I}$. Let $\Omega=\{a, b, c\}$ and $N=\{1,2,3,4,5,6\}$. The feasible set $\phi(S)=N$ for all coalitions $S$ except that $\phi(\{3\})=\{a, c\}$. The (single-peaked) preferences of the players are given by:

$$
\begin{aligned}
& v_{1}(a)=8, v_{1}(b)=3, v_{1}(c)=1 \\
& v_{2}(a)=45, v_{2}(b)=48, v_{2}(c)=0 \\
& v_{3}(a)=0, v_{3}(b)=50, v_{3}(c)=25 \\
& v_{4}(a)=50, v_{4}(b)=0, v_{4}(c)=0 \\
& v_{5}(a)=0, v_{5}(b)=0, v_{5}(c)=50 \\
& v_{6}(a)=0, v_{6}(b)=0, v_{6}(c)=50 .
\end{aligned}
$$

The function $h(\cdot)$ is given by

$$
h(1)=100, h(2)=102, h(3)=108, h(4)=116, h(5)=117, h(6)=118 .
$$

Suppose there is a Nash equilibrium $\left(\omega_{1}, \ldots, \omega_{6}\right)$. Then $\omega_{4}=a, \omega_{5}=\omega_{6}=c$ and $\omega_{2} \neq c$. 
Suppose that $\omega_{2}=b$. Then $\omega_{3}=b$ and the best response of player 1 is $a$. But $(a, b, b, a, c, c)$ is not a Nash equilibrium since player 2 would rather switch to $a$.

Suppose that $\omega_{2}=a$. Since player 1 would not choose $b$, player 3 must choose $c$. In this case player 1 will select $c$. But $(c, a, c, a, c, c)$ is not a Nash equilibrium since player 2 would rather switch to $b$. Thus, this environment does not admit a Nash equilibrium.

Even though the set of Nash equilibria is, in general, empty within the class $\mathcal{E}^{\prime}{ }_{I R}$, there exists an interesting subset of this class for which the existence of a Nash equilibrium can be rescued. As in Greenberg (1979), we require that all coalitions of the same size have identical feasible sets:

Anonymity of the feasible correspondence - AFC: For every two coalitions $S, S^{\prime}$ with $|S|=\left|S^{\prime}\right|$, we have $\phi(S)=\phi\left(S^{\prime}\right)$.

Then we have the following:

Proposition 5.2: Let $E \in \mathcal{E}_{\mathcal{I} \mathcal{R}}^{\prime}$, and suppose that AFC holds. Then there exists a Nash equilibrium.

Proof: See Appendix.

An important example of group formation game with increasing returns is the second best taxation game introduced by Guesnerie and Oddou (1981):

Example 5.3: There is an economy with $n$ agents and two goods, one private and one pure public good. The direct

preferences of player $i$ over consumption plans $\left(x_{i}, y\right) \in \Re_{+}^{2}$, where $x_{i}$ and $y$ her consumption of the private and public good, respectively, are represented by the utility function $U_{i}\left(x_{i}, y\right)$. Player $i$ has an initial endowment $w_{i}$ in private good and the public good is produced from the private good through a constant returns technology normalized to 1. Lump sum transfers cannot be used to finance public good production and the only of financing of 
production of the public good is through taxes proportional to initial endowments in private good. Two games can be considered depending on how $\Omega$ is defined. First is a taxation game, which was the focus of the Guesnerie and Oddou study), where players form groups according to the taxation rate $t \in[0,1]$ they select. The set of alternatives is $\Omega=[0,1]$, the utility functions are $u_{i}(\omega, S)=U_{i}\left((1-\omega) w_{i}, \omega \sum_{j \in S} w_{j}\right)$, and $\phi(S)=[0,1]$ for all $S \subseteq N$.

Alternatively, there is a production game, where players form groups according to the level of production $y$ of the public good they select. Then $\Omega=\Re_{+}$and $u_{i}(\omega, S)=U_{i}\left(w_{i}-\right.$ $\left.\omega \frac{w_{i}}{\sum_{j \in S} w_{j}}, \omega\right)$ and $\phi(S)=\left\{\omega \in \Re_{+}: \omega \leq \sum_{j \in S} w_{j}\right\}$ for each group $S$.

It is straightforward to show that from the point of view of strong Nash equilibria, the two games are equivalent. However, they are not when Nash equilibria are considered. In both games, $\mathrm{PE}$ is satisfied and $\mathrm{SP}$ is satisfied, too, whenever $U_{i}$ is quasi-concave. AN is satisfied only when the initial wealth is distributed uniformly, i.e., $w_{i}=w_{j}$ for all $i, j \in N$. Separability follows from separability assumptions on $U_{i}$.

Under the assumption of equal wealth, both games are in $\mathcal{E}$ and satisfy AFC. If, moreover, $U_{i}\left(x_{i}, y\right)=v_{i}\left(x_{i}\right)+g(y)$ for all $i \in N$, then both games belong to $\mathcal{E}^{\prime}$, by Proposition 5.2. both admit Nash equilibria. However, as demonstrated in Weber and Zamir (1985) and Konishi, Le Breton and Weber (1998), both games, which are equivalent in that respect, may fail to possess strong Nash equilibria.

If the initial wealth is not uniformly distributed across players, then, as demonstrated in Konishi, Le Breton and Weber (1998), the production game may fail to possess a Nash equilibrium, even if we assume quasi-linearity with respect to the private good i.e. $U_{i}\left(x_{i}, y\right)=$ $x_{i}+g_{i}(y)$ for all $i \in N$.

It is clear that the production game can be extended in many directions. One could consider the production of several public goods and assume their indivisibility. That would alter the nature of the problem and allow us to introduce effective groups, dichotomy of feasible correspondence and even exclusive effectiveness. But all these issues go beyond the 
scope of this paper and are left for future research.

Even though the existence of strong Nash equilibria is, in general, difficult to obtain, we show that the Euclidean preferences assumption PE yields the desirable existence:

Proposition 5.4: Within the class $\mathcal{E}^{\prime \prime}{ }_{I R}$, there exists a strong Nash equilibrium.

Proof: See Appendix.

As already pointed out in our examination of the Guesnerie and Oddou taxation and production games, the existence of a strong Nash equilibrium cannot be extended to the classes $\mathcal{E}_{I R}$ and $\mathcal{E}_{I R}^{\prime}$. However, the Greenberg and Weber (1993) result, mentioned in the beginning of this section, yields the existence of a strong Nash equilibrium within the class $\mathcal{E}_{I R}^{\prime}$, provided that the utility of every individual $i$ derived from a given alternative $\omega$ within any coalitions $S$ and $S^{\prime}$ is the same as long as $\omega$ is feasible for both $S$ and $S^{\prime}$, Formally,

Proposition 5.5 (Greenberg and Weber (1993): Suppose that the environment $E \in$ $\mathcal{E}_{I R}^{\prime}$ is such that the utility function of individual $i$ is represented by

$$
u_{i}(\omega, S)=\left\{\begin{array}{lll}
v_{i}(\omega) & \text { if } \quad \omega \notin \phi(S) \\
v_{i}(\omega)+h & \text { if } \quad \omega \in \phi(S)
\end{array}\right.
$$

where $v_{i}(\cdot)$ is single-peaked over $\phi(S)$, and the number $h$ is such that for every $\omega, \omega^{\prime} \in \Omega$ $h>v_{i}(\omega)-v_{i}\left(\omega^{\prime}\right)$. Then $E$ admits a strong Nash equilibrium.

\section{Decreasing Returns}

In this section we consider the case of decreasing returns. The assumption DR of decreasing returns should not be confused with the assumption of negative externality, stating that $u_{i}(\omega, S) \geq u_{i}\left(\omega, S^{\prime}\right)$ for every $i \in N$, every two coalitions with $i \in S \subset S^{\prime}$ and every $\omega \in \phi(S) \cap \phi\left(S^{\prime}\right)$. This is the opposite of the positive externality assumption maintained through this paper. ${ }^{10}$.

\footnotetext{
${ }^{10}$ Under negative externality, anonymity and constant returns, there always exists a strong Nash equilibrium in the group formation game (Milchtaich (1996), Konishi, Le Breton and Weber (1997d)
} 
The assumption of decreasing returns means something different as every player benefits from a given alternative to be chosen by a larger group. To provide some intuition, assume that each player $i$ has a predetermined list $\phi(\{i\})$ of conceivable or acceptable alternatives. Then the set of possible compromises $\phi(S)$ for group $S$ is the intersection of all individually acceptable choices of its members $\phi(S)=\cap_{i \in S} \phi(\{i\})$. With that interpretation of feasibility, large coalitions may find difficult to reach a conceivable compromise. Then to look for equilibria simply amounts to search among the partitions of $N$ into conceivable groups, where group is conceivable if its feasible set is nonempty. The conflict here is obvious: everybody wants to be in a large group - it is just difficult to reach a compromise there.

The result in Konishi, Le Breton and Weber (1997a) obtained under constant returns implies that, within the class $\mathcal{E}_{D R}$, a Nash equilibrium may fail to exist. However, we are able to show the existence of a Nash equilibrium for all environments in the class $\mathcal{E}^{\prime}{ }_{D R}$.

Proposition 6.1: Within the class $\mathcal{E}^{\prime}{ }_{D R}$, there always exists a Nash equilibrium.

\section{Proof: See Appendix.}

As far as a strong Nash equilibrium is concerned, it turns out that even within the class the class $\mathcal{E}^{\prime \prime}{ }_{D R}$ it may fail to exist. Consider the following example:

Example 6.2: We construct a quadruple $E$ that belongs to $\mathcal{E}^{\prime \prime}{ }_{D R}$. Let $\Omega=\{a, b, c\}$ and $N=\{1,2,3\}$. The alternatives $a, b, c$, that represent the ideal points of individuals $1,2,3$, respectively, are located on the line. Let $a=b-1=c-2$. The feasible set $\phi(\{i\})=N$ for all singletons, $\phi(\{1,3\})=\{a, c\}, \phi(\{1,2\})=\phi(\{2,3\})=\{b\}$ and $\phi(N)$ is empty. The function $h(\cdot)$ is given by $h(1)=3, h(2)=6, h(3)=7$.

Suppose there is a strong Nash equilibrium $\left(\omega_{1}, \omega_{2}, \omega_{3}\right)$. There are five possible candidates: $(b, b, c)$ - blocked by coalition $(1,3)$ via alternative $a$.

$(a, b, b)$ - blocked by coalition $(1,3)$ via alternative $c$.

$(a, b, a)$ - blocked by coalition $(2,3)$ via alternative $c$. 
$(c, b, c)$ - blocked by coalition $(1,2)$ via alternative $a$.

$(a, b, c)$ - blocked by coalition $(1,2)$ via alternative $a$.

Thus, there exists no strong Nash equilibrium.

The existence of a strong Nash equilibrium can be rescued if we impose some additional conditions on the feasible correspondence and feasible sets. First, we require that every individually feasible set $\phi(\{i\})$ is nonempty and is consecutive in the sense that for every three alternatives $\omega, \omega^{\prime}, \omega^{\prime \prime}$ with $\omega<\omega^{\prime}<\omega^{\prime \prime}, \omega, \omega^{\prime \prime} \in \phi(\{i\})$ imply $\omega^{\prime} \in \phi(\{i\})$. The consecutiveness will be also imposed across feasible sets by requiring that an alternative that belongs to the feasible sets for two individuals, should be feasible for all intermediate individuals between these two. Formally,

Consecutiveness of the Feasibility Correspondence - CFC: (i) For every individual $i$, the set $\phi(\{i\})$ is a consecutive subset of $\Omega$ containing $i$ 's ideal point $\omega^{i}$;

(ii) For every three individuals $i<j<k$ and an alternative $\omega \in \phi(\{i\}) \cap \phi(\{k\})$, it follows that $\omega \in \phi(\{j\})$;

(iii) The feasible set of every coalition $S \subset N$ is the intersection of the individual feasible sets of all of its members: $\phi(S)=\bigcap_{i \in S} \phi(\{i\})$.

Then

Proposition 6.3: If $\mathrm{CFC}$ holds then every environment within the class $\mathcal{E}^{\prime \prime}{ }_{D}$, admits a strong Nash equilibrium.

Proof: See Appendix.

\section{Appendix}

Proof of Proposition 3.4: It is straightforward to show that the statement in Proposition 3.4 follows from the fact that under the assumptions that $E$ is a society satisfying 
DFA,PE and IR, a strategy profile $x=\left(\omega_{1}, \ldots, \omega_{n}\right)$ is a strong Nash equilibrium if and only if there is no coalition $S \subset N$ and an alternative $\omega \in \phi(S)$ such that $u_{i}(\omega, S)>u_{i}\left(\omega_{i}, N_{\omega_{i}}(x)\right.$ for all $i \in S$.

First, let $x=\left(\omega_{1}, \ldots, \omega_{n}\right)$ be a strong Nash equilibrium. We have to show that there is no coalition $S \subset N$ and an alternative $\omega \in \phi(S)$ such that $u_{i}(\omega, S)>u_{i}\left(\omega_{i}, N_{\omega_{i}}(x)\right)$ for all $i \in S$. Assume, in negation, that there is a coalition $S \subset N$ and an alternative $\omega \in \phi(S)$ with $u_{i}(\omega, S)>u_{i}\left(\omega_{i}, N_{\omega_{i}}(x)\right)$ for all $i \in S$. Let $x^{\prime}=\left(\omega_{1}^{\prime}, \ldots, \omega_{n}^{\prime}\right)$ be such that $\omega_{i}^{\prime}=\omega$ for all $i \in S$ and $\omega_{i}^{\prime}=\omega_{i}$ for all $i \notin S$. By construction and IR, it follows that $N_{\omega}\left(x^{\prime}\right) \supseteq S$. Since, by positive externality, $u_{i}\left(\omega, N_{\omega}\left(x^{\prime}\right)\right) \geq u_{i}(\omega, S)>u_{i}\left(\omega_{i}, N_{\omega_{i}}(x)\right)$ for all $i \in S$, we contradict our assumption that $x=\left(\omega_{1}, \ldots, \omega_{n}\right)$ is a strong Nash equilibrium.

Second, let $x=\left(\omega_{1}, \ldots, \omega_{n}\right)$ be a strategy profile such that there is no coalition $S \subset N$ and an alternative $\omega \in \phi(S)$ with $\left.u_{i}(\omega, S)>u_{i}\left(\omega_{i}, N_{\omega_{i}}(x)\right)\right)$ for all $i \in S$. We have to prove that $x$ is a strong Nash equilibrium. Assume, on the contrary, that there exists $x^{\prime}=\left(\omega_{1}^{\prime}, \ldots, \omega_{n}^{\prime}\right)$ such that $\omega_{i}^{\prime}=\omega_{i}$ for all $i \notin S$ and $u_{i}\left(\omega_{i}^{\prime}, N_{\omega_{i}^{\prime}}\left(x^{\prime}\right)\right)>u_{i}\left(\omega_{i}, N_{\omega_{i}}(x)\right)$ for all $i \in S$. Note that $\omega_{i}^{\prime} \in\left\{\omega_{1}, \ldots, \omega_{n}\right\}$ for all $i \in S$. Otherwise, our original assertion would be violated via the group $N_{\omega_{i}^{\prime}}\left(x^{\prime}\right)$ choosing the alternative $\omega_{i}^{\prime}$. This implies that the size of one of the existing groups, say, the group containing player $j$, has strictly increased. That is, $N_{\omega_{j}}\left(x^{\prime}\right) \supset N_{\omega_{j}}(x)$ and, in particular, $S \cap N_{\omega_{j}}\left(x^{\prime}\right) \neq \emptyset$. By construction, $u_{i}\left(\omega_{j}, N_{\omega_{j}}\left(x^{\prime}\right)\right)>u_{i}\left(\omega_{i}, N_{\omega_{i}}(x)\right)$ for all $i \in S \cap N_{\omega_{j}}\left(x^{\prime}\right)$ and, by PE, $u_{i}\left(\omega_{j}, N_{\omega_{j}}\left(x^{\prime}\right)\right)>u_{i}\left(\omega_{i}, N_{\omega_{i}}(x)\right)$ for all $i \in N_{\omega_{j}}\left(x^{\prime}\right) \backslash S$. Therefore, $u_{i}\left(\omega, N_{\omega_{j}}\left(x^{\prime}\right)\right)>u_{i}\left(\omega_{i}, N_{\omega_{i}}(x)\right)$ for all $i \in N_{\omega_{j}}\left(x^{\prime}\right)$ with $\omega \equiv \omega_{j}$, a contradiction.

Proof of Proposition 4.6: If the family $\mathcal{C}$ satisfies exclusive effectiveness, then either $\phi(S)=\emptyset$ or $\phi(N \backslash S)=\emptyset$. Therefore, the condition of bilateral agreement is equivalent to the following: for every $i$ and $S \in \mathcal{S}^{\prime}$ and $\phi(S)=\Omega$, there exists $\omega \in \phi(N)$ such that $\max _{\omega^{\prime} \in \Omega} U_{i}\left(\omega^{\prime}, S\right) \leq U_{i}(\omega, N)$. But the last inequality is guaranteed by $\mathrm{PE}$.

Now let $m \leq \nu(\phi)-2$. The continuous version of the Nakamura's theorem ${ }^{11}$ implies that

\footnotetext{
${ }^{11}$ The first version of the Nakamura's theorem is due to Greenberg (1979) who studied families of effective
} 
the core of the NTU cooperative game $\bar{v}$, defined by ${ }^{12}$ :

$$
\bar{v}(S)=\left\{u \in \Re^{n}: \exists \omega \in \phi(S) \text { s. t. } u_{i} \leq U_{i}(\omega, N) \forall i \in S\right\}
$$

is nonempty. Let $u$ be an element of the core and $\omega \in \phi(N)$ be such that $u=U_{i}(\omega, N)$ for all $i \in N$. We claim that the profile $(\omega, \omega, \ldots, \omega)$ is a strong Nash equilibrium.

Suppose it is not. Then there exists a coalition $S$ and $\omega^{\prime} \in \phi(S)$ such that $U_{i}\left(\omega^{\prime}, S\right)>$ $U_{i}(\omega, N)$ for all $i \in S$. From the positive externality assumption, we deduce that $U_{i}\left(\omega^{\prime}, N\right)>$ $U_{i}(\omega, N)$ for all $i \in S$. Let $u^{\prime} \in \Re^{n}$ be defined as follows: $u_{i}^{\prime}=U_{i}\left(\omega^{\prime}, N\right)$ for all $i \in S$ and $u_{i}^{\prime}=u_{i}$ for all $i \notin S$. By construction, $u^{\prime} \in \bar{v}(S)$, but since $u_{i}^{\prime}>u_{i}$ for all $i \in S$, it contradicts the assumption that $u$ is in the core of $\bar{v}$.

Proof of Proposition 5.2: The proof is carried out by using the potential function approached pioneered by Rosenthal (1973). (See also Monderer and Shapley (1996), Konishi, Le Breton and Weber (1997a)).

AFC implies that for every $\omega$ there exists a positive integer $n(\omega)$ such that $\omega \in \phi(S)$ for every $S$ with $|S| \geq n(\omega)$ and $\omega \notin \phi(S)$ for every $S$ with $|S|<n(\omega)$.

Consider the function $\Psi$ defined on the set of all strategy profiles:

$$
\Psi(x)=\sum_{\omega \in \Omega}\left[\sum v_{i}(\omega)+\sum_{k=n(\omega)}^{\left|N_{x}(\omega)\right|} h(k)\right]
$$

(If $n(\omega)>\left|N_{x}(\omega)\right|$ then the corresponding sum is set to zero.)

Take a maximum of the function $\Psi, x=\left(\omega_{1}, \ldots \omega^{n}\right)$. We shall show that $x$ is a Nash equilibrium. First, there is at least one $i$ such that $\omega_{i} \in \phi\left(N_{x}\left(\omega_{i}\right)\right)$. Otherwise, DFA implies that all players choosing the same alternative would generate a higher value of function $\Psi$. Moreover, DFA implies that $\omega_{i} \in \phi\left(N_{x}\left(\omega_{i}\right)\right)$ for each $i$. Suppose now that there is a player $i$ coalitions $\mathcal{C}$ satisfying AFC. The extension to an arbitrary $\mathcal{C}$ is due to Le Breton (1987), Schofield (1984) and Strnad(1985). It should be pointed out that in this setting existence of the core does not follow in general from a balancedness argument, as the game is not always balanced (Le Breton (1989)).

${ }^{12}$ It should be noted that the game $\bar{v}$ is different from the game $v$ defined earlier. 
who wishes to switch from $\omega_{i}$ to $\omega$. Then $\omega \in \phi\left(N_{x}(\omega) \cup\{i\}\right)$ and

$$
v_{i}\left(\omega_{i}\right)+h\left(\left|N_{x}\left(\omega_{i}\right)\right|\right)<v_{i}(\omega)+h\left(\left|N_{x}(\omega)\right|+1\right) .
$$

This would imply that $\Psi(x)<\Psi\left(\left(x_{-i}, \omega\right)\right)$, where $\left(x_{-i}, \omega\right)$ is the profile with $i$ choosing $\omega$ and all other players choosing the same strategies as in $x$. But this is a contradiction to $x$ being a maximum of the function $\Psi$.

Proof of Proposition 5.4: Assume that all players are ordered with respect to their ideal points so that $\omega^{1} \leq \omega^{2} \leq \ldots \leq \omega^{n}$.

By Greenberg and Weber (1986), there exists a strategy profile $x$ such that every coalition in $P(x)$ is consecutive and there is no consecutive coalition $S$ and $\omega \in \phi(S)$ such that

$$
-\left|\omega^{i}-\omega\right|+h(|S|)>-\left|\omega^{i}-\omega_{i}\right|+h\left(\left|N_{x}\left(\omega_{i}\right)\right|\right) \text { for all } i \in S \text {. }
$$

We shall say then that $S$ blocks $x$.

In order to show that $x$ is a strong Nash equilibrium, it remains to demonstrate that there is no nonconsecutive coalition $S$ and $\omega \in \phi(S)$ such that

$$
-\left|\omega^{i}-\omega\right|+h(|S|)>-\left|\omega^{i}-\omega_{i}\right|+h\left(\left|N_{x}\left(\omega_{i}\right)\right|\right) \text { for all } i \in S \text {. }
$$

Suppose, in negation, that there is a nonconsecutive coalition $S$ that blocks $x$. Nonconsecutiveness of $S$ implies that there exist three players $i<j<k$ such that $i$ and $j$ belong to $S$ whereas $j$ does not. That is, there is an alternative $\omega$ such that

$$
\begin{gathered}
-\left|\omega^{i}-\omega\right|+h(|S|)>-\left|\omega^{i}-\omega_{i}\right|+h\left(\left|N_{x}\left(\omega_{i}\right)\right|\right) \\
-\left|\omega^{k}-\omega\right|+h(|S|)>-\left|\omega^{k}-\omega_{k}\right|+h\left(N_{x}\left(\mid \omega_{k}\right) \mid\right) .
\end{gathered}
$$

Let us introduce the degree of nonconsecutiveness $n(S)$ of a coalition $C$. Let $m(C)$ be its lowest member and $M(C)$ the highest. Then $n(C)$ represents the maximal size of a consecutive coalition $C^{\prime}$ such that $C^{\prime} \subset\{m(C), \ldots, M(C)\}$ and $C^{\prime} \cap C=\emptyset$. Obviously $n(C)>0$ if and only $C$ is nonconsecutive. 
Assume, without loss of generality, that (i) the degree of nonconsecutiveness of $S$ is the lowest among nonconsecutive coalitions that block $(P, A)$, (ii) $|S|=\min _{C \mid n(C)=n(S)}|C|$, and (iii) $k-i=n(S)+1$.

Note then that neither the coalition $N_{x}\left(\omega_{j}\right) \cup\{i\}$ nor the coalition $N_{x}\left(\omega_{j}\right) \bigcup\{k\}$ can block $x$ as both $n\left(N_{x}\left(\omega_{j}\right) \bigcup\{i\}\right)$ and $n\left(N_{x}\left(\omega_{j}\right) \bigcup\{k\}\right)$ are smaller than $n(S)$. In particular, they cannot use $\omega_{j}$ in order to block. By DFA and PE, we have

$$
\begin{aligned}
& -\left|\omega^{i}-\omega_{j}\right|+h\left(\left|N_{x}\left(\omega_{j} \mid+1\right) \leq-\right| \omega^{i}-\omega_{i} \mid+h\left(\mid N_{x}\left(\omega_{i} \mid\right)\right.\right. \\
& -\left|\omega^{k}-\omega_{j}\right|+h\left(\left|N_{x}\left(\omega_{j} \mid+1\right) \leq-\right| \omega^{k}-\omega_{k} \mid+h\left(\mid N_{x}\left(\omega_{k} \mid\right) .\right.\right.
\end{aligned}
$$

Combining (1) - (4) we have

$$
\begin{aligned}
& -\left|\omega^{i}-\omega_{j}\right|+h\left(\left|N_{x}\left(\omega_{j} \mid+1\right)<-\right| \omega^{i}-\omega \mid+h(|S|)\right. \\
& -\left|\omega^{k}-\omega_{j}\right|+h\left(\left|N_{x}\left(\omega_{j} \mid+1\right)<-\right| \omega^{k}-\omega \mid+h(|S|) .\right.
\end{aligned}
$$

The inequalities (5) and (6) imply that

$$
-\left|\omega^{j}-\omega_{j}\right|+h\left(\left|N_{x}\left(\omega_{j} \mid+1\right)<-\right| \omega^{j}-\omega \mid+h(|S|)\right.
$$

Let $T=S \bigcup\{j\}$. Since DFA implies $\omega \in \phi(T)$. and $h$ is increasing, it follows that $T$ blocks $x$, a contradiction. Indeed, if $T$ is consecutive, by our assumption, it cannot block $x$. If $T$ is nonconsecutive then $n(T) \leq n(S)$, whereas $|S|<|T|$, contrary to our choice of $S$. $\square$

Proof of Proposition 6.1: We again apply the potential functions approach. Consider the function $\Psi$ defined on the set of all strategy profiles:

$$
\Psi(x)=\sum_{\omega \in \Omega}\left[\sum v_{i}(\omega)+\lambda(x, \omega)\right]
$$

where

$$
\lambda(x, \omega)=\left\{\begin{array}{lll}
0 & \text { if } \quad \omega \notin \phi\left(N_{x}(\omega)\right) \\
\sum_{k=1)}^{\left|N_{x}(\omega)\right|} h(k) & \text { if } \quad \omega \in \phi\left(N_{x}(\omega)\right)
\end{array}\right.
$$


Let $x$ be a maximum of the function $\Psi$. If it is not a Nash equilibrium, there is an individual $i$ that wishes to switch from $a$ to $b$. In this case, the alternative $b$ must be feasible for coalition $N_{x}(b) \bigcup\{i\}$.

There could not be the case where individual $i$ is assigned to the group $N_{x}(a)$ choosing alternative $a$ outside its feasible set, while in the same time there an alternative $b$ such that $b \in \phi\left(N_{x}(b)\right) \bigcup\{i\}$. Moreover, $a$ must be feasible for $N_{x}(a)$, otherwise the reassignment of $i$ from $a$ to $b$ would have increased the value of the function $\Psi$, a contradiction to $x$ being its maximum. But then

$$
\Psi\left(x_{i}, b\right)-\Psi(x)=v_{i}(b)+h\left(\left|N_{x}(b)\right|+1\right)-v_{i}(a)-h\left(\left|N_{x}(a)\right|\right)>0,
$$

a contradiction to $x$ being a maximum.

Proof of Proposition 6.3: As in the proof of Proposition 5.4, consider a strategy profile $x$ such that every coalition in $P(x)$ is consecutive and there is no consecutive coalition $S$ that blocks $x$. We shall show that there is no nonconsecutive coalition that blocks $x$.

Suppose, in negation, that there is a nonconsecutive coalition $S$ that blocks $x$. Again, there are three players $i<j<k$ such that $i$ and $j$ belong to $S$ whereas $j$ does not. There is $\omega$ such that

$$
\begin{array}{r}
-\left|\omega^{i}-\omega\right|+h(|S|)>-\left|\omega^{i}-\omega_{i}\right|+h\left(\left|N_{x}\left(\omega_{i}\right)\right|\right) \\
-\left|\omega^{k}-\omega\right|+h(|S|)>-\left|\omega^{k}-\omega_{k}\right|+h\left(\left|N_{x}\left(\omega_{k}\right)\right|\right) .
\end{array}
$$

Again, assume, without loss of generality, that (i) the degree of nonconsecutiveness of $S$ is the lowest among nonconsecutive coalitions that block $(P, A)$, (ii) $|S|=\min _{C \mid n(C)=n(S)}|C|$, and (iii) $k-i=n(S)+1$.

Note then that neither the coalition $N_{x}\left(\omega_{j}\right) \bigcup\{i\}$ nor the coalition $N_{x}\left(\omega_{j}\right) \bigcup\{k\}$ can block $x$ by using $\omega_{j}$, as both $n(S(j) \bigcup\{i\})$ and $n(S(j) \bigcup\{k\})$ are smaller than $n(S)$. Here we have to 
deviate from the proof of the Proposition 5.4. The fact that one of the two coalitions may not block $x$, could be due to the feasibility constraints. In other words, if $\omega_{j} \in \phi\left(N_{x}\left(\omega_{j}\right) \bigcup\{i\}\right)$ and $\omega_{j} \in \phi\left(N_{x}\left(\omega_{j}\right) \bigcup\{k\}\right)$, then we can complete our proof as before.

Suppose therefore that $\omega_{j} \notin \phi\left(N_{x}\left(\omega_{j}\right) \bigcup\{i\}\right)$. Since $i$ and $k$ can co-exist in coalition $S$, the intersection of $\phi(\{i\})$ and $\phi(\{k\})$ is nonempty. Since both sets are consecutive it follows that $\omega_{j} \in \phi(\{k\})$. Thus, the fact that $N_{x}\left(\omega_{j}\right) \bigcup\{k\}$ cannot block $x$ by using $\omega_{j}$, is not due to feasibility and

$$
-\left|\omega^{k}-\omega_{j}\right|+h\left(\left|N_{x}\left(\omega_{j}\right)\right|+1\right) \leq-\left|\omega^{k}-\omega_{k}\right|+h\left(\left|N_{x}\left(\omega_{k}\right)\right|\right)
$$

Combining (9) and (10), we have

$$
-\left|\omega^{k}-\omega_{j}\right|+h\left(\left|N_{x}\left(\omega_{j}\right)\right|+1\right)<-\left|\omega^{k}-\omega\right|+h(|S|) .
$$

However, the alternative $\omega$ is feasible for $S \bigcup\{j\}$. But, by our assumptions, this coalition could not block $x$ and we have:

$$
-\left|\omega^{j}-\omega_{j}\right|+h\left(\left|N_{x}\left(\omega_{j}\right)\right|\right) \geq-\left|\omega^{j}-\omega\right|+h(|S|+1)
$$

Inequalities (11) and (12) imply that

$$
\left|\omega^{k}-\omega_{j}\right|-\left|\omega^{k}-\omega\right|>\left|\omega^{j}-\omega_{j}\right|-\left|\omega^{j}-\omega\right|
$$

Since $\omega^{k} \geq \omega^{j}$ it follows that $\omega_{j}<\omega$. However, since $\omega^{i} \leq \omega^{j}$ and $\omega \in \phi(\{i\})$, it follows that $\omega_{j} \in \phi(\{i\})$ as well. But it would contradict the fact that $\omega_{j}$ is not feasible for the coalition $N_{x}\left(\omega_{j}\right) \bigcap\{i\}$

\section{References}

Aumann, R.J. (1959) "Acceptable points in general cooperative $n$-person games", in Contributions to the Theory of Games, Vol. IV, Tucker, A.W. and R.D. Luce, eds., Princeton University Press, Princeton, 1959. 
Aumann, R.J. and J. Drèze (1974) "Cooperative games with coalition structure", International Journal of Game Theory 3, 217-237.

Banerjee, S., Konishi, H. and T. Sömnez (2001) "Core in a simple coalition formation game", Social Choice and Welfare 18, 135-153.

Belleflamme, P. (2000) "Stable coalition structures with open membership and asymmetric firms", Games and Economic Behavior 30, 1-21.

Bernheim, D., Peleg, B. and M.D. Whinston (1987) "Coalition-proof Nash equilibria: I concepts", Journal of Economic Theory 42, 1-12.

Bloch, F. (1995) "Endogenous structures of association in oligopolies", Rand Journal of Economics, 26, 537-556.

Bloch, F. (1996) "Sequential formation of coalitions with fixed payoff division and externalities", Games and Economic Behavior 14, 90-123.

Bloch, F. (1997) "Non-cooperative models of coalition formation in games with spillovers, in New Directions in the Economic Theory of the Environment, Carraro, C. and D. Siniscalco, eds., Cambridge University Press, Cambridge, 1997.

Bogomolnaia, A. and M.O. Jackson (2002) "The stability of hedonic coalition structures", Games and Economic Behavior 18, 201-230.

Deb, R., Weber, S. and E. Winter (1996) "The Nakamura theorem for coalition structures of quota games", International Journal of Game Theory 25, 189-198.

Demange, G. (1994) "Intermediate preferences and stable coalition structures", Journal of Mathematical Economics 23, 45-58.

Demange, G. and R. Guesnerie (1997) "Nonemptiness of the core: low multidimensional decision spaces and one-dimensional preferences", Research in Economics 51, 7-18.

Drèze, J. and J. Greenberg (1980) "Hedonic coalitions: optimality and stability", Econometrica, 48, 987-1003.

Greenberg, J. (1979) "Consistent majority rules over compact sets of alternatives", 
Econometrica 47, 627-636.

Greenberg, J. (1994) "Coalition Structures", in Handbook of Game Theory with Applications, Vol. 2, Aumann, R. and S. Hart, eds., North Holland, Amsterdam.

Greenberg, J. and S. Weber (1982) "The equivalence of superadditivity and balancedness in a tax proportional game", Economic Letters 9, 113-117.

Greenberg, J. and S. Weber (1986) "Strong Tiebout equilibrium under restricted preferences domain", Journal of Economic Theory 38, 101-117.

Greenberg, J. and S. Weber (1993) "Stable coalition structures with

unidimensional set of alternatives", Journal of Economic Theory 60, 693-703.

Greenberg, J. and S. Weber (1994) "Stable coalition structures in consecutive games", in Frontiers in Game Theory, Binmore, K., Kirman, A. and P. Tani, eds., MIT Press, Cambridge.

Guesnerie, R. (1995) A Contribution to the Pure Theory of Taxation, Cambridge University Press, Cambridge.

Guesnerie, R. and C. Oddou (1979) "On economic games which are not necessarily superadditive", Economic Letters 3, 301-306.

Guesnerie, R. and C. Oddou (1981) "Second best taxation as a game", Journal of Economic Theory 25, 67-91.

Guesnerie, R. and C. Oddou (1988) "Increasing returns to size and their limits", Scandinavian Journal of Economics 90, 259-273.

Kaneko, M. and M.H. Wooders (1982) "Cores of Partitioning games", Mathematical Social Sciences 3, 313-327.

Konishi, H. and P.C. Fishburn (1996) "Quasi-linear utility in a discrete choice model", Economic Letters, 51, 197-200.

Konishi, H., Le Breton, M. and S. Weber (1997a) "Pure strategy Nash equilibria in a group formation game with positive externalities", Games and Economic Behavior 21, 
161-182.

Konishi, H., Le Breton, M. and S. Weber (1997b) "Free mobility equilibrium in a local public goods economy with congestion", Research in Economics 51, 19-30.

Konishi, H., Le Breton, M. and S. Weber (1997c) "Equivalence of strong and coalitionproof Nash equilibria in games without spillovers", Economic Theory 9, 97-113.

Konishi, H., Le Breton, M. and S. Weber (1997d) "Equilibrium in a model with partial rivalry", Journal of Economic Theory 72, 225-237.

Konishi, H., Le Breton, M. and S. Weber (1997e) "Group formation in games without spillovers: a noncooperative game-theoretical approach", in New Directions in the Economic Theory of the Environment, Carraro, C. and D. Siniscalco, eds., Cambridge University Press, Cambridge.

Konishi, H., Le Breton, M. and S. Weber (1998) "Equilibrium in a finite local public goods economy", Journal of Economic Theory 79, 224-244.

Le Breton, M (1987) "On the core of voting games", Social Choice and Welfare 4, 295-305.

Le Breton, M. (1989) "A note on balancedness and nonemptiness of the core of voting games", International Journal of Game Theory 18, 111-117.

Le Breton, M., Owen, G. and S. Weber (1992) "Strongly balanced cooperative games", International Journal of Game Theory 20, 419-427.

Le Breton, M. and S. Weber (1995) "Stability of coalition structures and the principle of optimal partitioning", in Social Choice, Welfare and Ethics, Barnett,W., Moulin, H., Salles, M. and N. Schofield, eds., Cambridge University Press, Cambridge.

Milchtaich, I. (1996) "Congestion games with player-specific payoff functions", Games and Economic Behavior 13, 124-143.

Milchtaich, I. and E. Winter (2002) "Stability and segregation in group formation", Games and Economic Behavior 38, 318-346.

Monderer, D. and L.S. Shapley (1996) "Potential games", Games and Economic Behavior 
$14,124-143$.

Nakamura, K. (1979) "The vetoers in a simple game with ordinal preferences", International Journal of Game Theory, 8, 55-61.

Ray, D. and R. Vohra (2001) "Coalitional power and public goods", Journal of Political Economy 109, 1355-1384.

Rosenthal, R.W. (1973) "A class of games possessing a pure-strategy Nash equilibrium", International Journal of Game Theory 2, 65-67.

Schofield, N. (1984) "Social equilibrium and cycles on compact sets", Journal of Economic Theory 33, 59-71.

Strnad, J. (1985) "The structure of continuous-valued neutral monotonic social choice functions", Social Choice and Welfare 2, 181-195.

Weber, S. and S. Zamir (1985) "Proportional taxation: nonexistence of stable structures in an economy with a public good", Journal of Economic Theory 35, 178-185.

Yi, S.S. (1997) "Stable coalition structures with externalities", Games and Economic Behavior 20, 201-237. 